\title{
Ocular Melanoma Presenting as Masquerade Syndrome
}

\author{
$\underline{\text { Sara Simões Macedo }}^{1}$, Mónica Teixeira $^{1}$, Andreia Correia $^{1}$, António Novais $^{1}$, Cátia Cabral $^{2}$ \\ ${ }^{1}$ Department of Internal Medicine, Centro Hospitalar Tondela-Viseu, Viseu, Portugal \\ ${ }^{2}$ Department of Internal Medicine, Hospital Trofa Vila Real, Vila Real, Portugal
}

Received: $12 / 04 / 2019$

Accepted: 28/05/2019

Published: 01/07/2019

How to cite this article: Simões Macedo S, Teixeira M, Correia A, Novais A, Cabral C. Ocular melanoma presenting as masquerade syndrome. EJCRIM 2019;6: doi:10.12890/2019_001118.

Acknowledgements: The authors wish to acknowledge Francisco Oliveira for help with English language.

Conflicts of Interests: The Authors declare that there are no competing interests.

This article is licensed under a Commons Attribution Non-Commercial 4.0 License

\section{ABSTRACT}

A masquerade syndrome is an ophthalmological entity where a neoplasm mimics an inflammatory condition. Ocular melanoma (chiefly uveal) may present with symptoms suggestive of intraocular inflammation such as endogenous endophthalmitis. Ocular melanoma is most commonly found in middle-aged and older caucasian patients. One-third of all uveal melanoma cases present asymptomatically. Early diagnosis facilitates treatment before ocular melanoma reaches metastatic stage IV. Current therapy options for stage IV patients are palliative care and clinical trial participation.

\section{LEARNING POINTS}

- Masquerade syndrome and intraocular haemorrhage can hide a neoplastic aetiology.

- A biopsy should be carried out for correct diagnosis if intraocular haemorrhage is present and a neoplastic pathology is suspected.

- A multidisciplinary approach should be adopted to enhance the quality of life of patients with metastatic ocular melanoma.

\section{KEYWORDS}

Ocular melanoma, intraocular haemorrhage, endogenous endophthalmitis, masquerade syndrome, metastasis

\section{CASE DESCRIPTION}

A 75-year-old woman presented with an 8-day history of pain, eye tearing and loss of vision in the right eye. There was no history of trauma or ocular surgery. On examination, there was periorbital oedema and erythema, exuberant chemosis, the cornea was transparent and a haemorrhage was seen in the anterior chamber. Right ocular echography revealed dense echoes with low mobility in the vitreous cavity (Fig. 1). The patient was admitted for aetiological study of endophthalmitis and began antibiotic therapy with ceftazidime and ophthalmic ciprofloxacin. There was progressive clinical improvement with topical and systemic antibiotics, ophthalmic dexamethasone, timolol, dorzolamide and methylprednisolone. The fasting blood sugar was $82 \mathrm{mg} / \mathrm{dl}$, while retroviral and autoimmune screening was negative. Systemic infection was excluded and cultures yielded no growth. Due to the inconclusive aetiological study of the endogenous endophthalmitis, the patient was referred for consultations with Internal Medicine and Ophthalmology clinicians.

A few months later, the patient represented with asthenia, anorexia and night sweats. On examination, there was hepatomegaly with several palpable nodules. A computed tomography (CT) scan of the thorax, abdomen and pelvis revealed pulmonary metastasis and hepatomegaly with a metastatic lesion. A CT-guided liver biopsy was performed without complications. Histological examination revealed metastatic melanoma. 
In view of the clinical evolution, the endogenous endophthalmitis and the metastatic melanoma, and after exclusion of other locations for this tumour, a presumptive diagnosis of stage IV ocular melanoma was made. The patient complained of irruptive pain, which was managed with fentanyl patches and oral fentanyl. Due to marked worsening of the patient's general condition, it was not possible to administer palliative chemotherapy. The patient rejected a proposal to participate in a clinical trial.

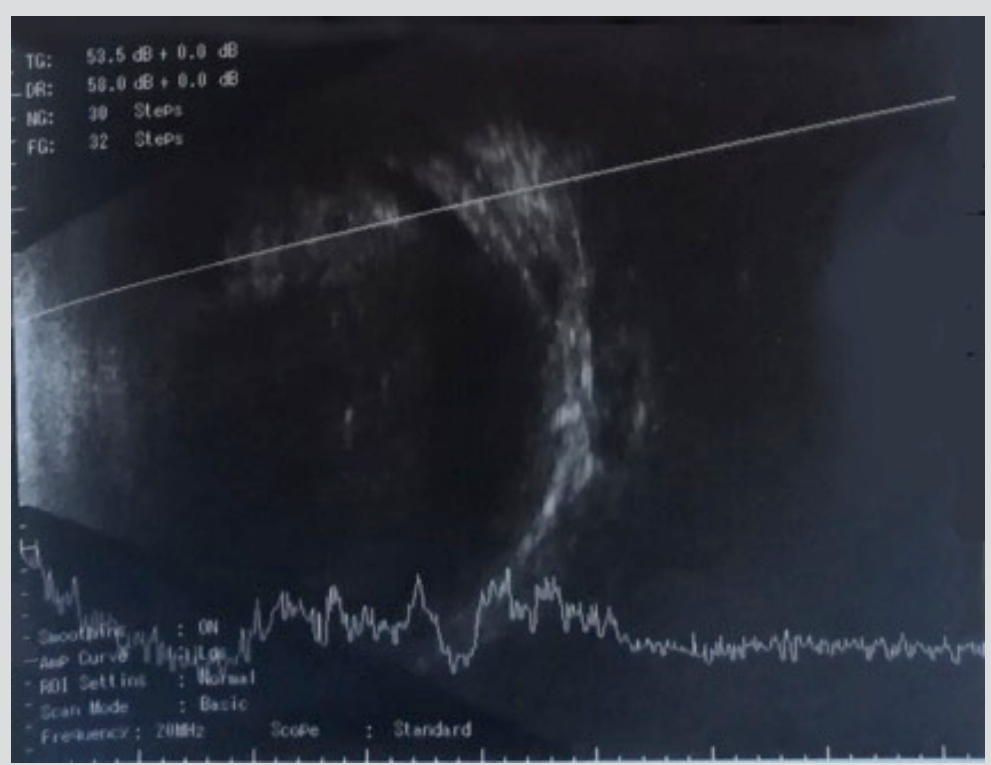

Figure 1. Ocular ultrasound suggesting endophthalmitis

\section{DISCUSSION}

Uveal melanomas are rare tumours that most frequently masquerade as inflammation ${ }^{[1]}$. The term masquerade syndrome first appeared in the ophthalmic literature in 1967 when it was used to describe conjunctival carcinoma presenting as chronic conjunctivitis ${ }^{[1]}$. Masquerade syndromes have since been defined as entities, including neoplastic processes, that mimic inflammatory conditions ${ }^{[1]}$.

Haemorrhage in the anterior chamber may be the initial clinical sign of uveal melanoma ${ }^{[2,3]}$. As highlighted in this case, an intraocular haemorrhage and endogenous endophthalmitis suggest a neoplastic pathology (uveal melanoma). A non-invasive diagnosis can usually be made based on ultrasonographic and funduscopic findings ${ }^{[1,4]}$. In a case with a high suspicion of neoplastic pathology, a fine needle diagnostic biopsy must be performed to distinguish haemorrhage from ocular melanoma, metastatic tumour or other lesions ${ }^{[5]}$. This technique can also be employed for molecular prognostic testing and is useful for stratifying high-risk patients into clinical trials ${ }^{[4,5]}$.

Without an early diagnosis and treatment, ocular melanoma may progress to metastatic stage IV. The most common initial sites of melanoma metastasis include the liver, lung, skin, soft tissue and bone ${ }^{[4]}$. At this stage, the principal role of the clinician is to improve the quality of life of patients by controlling their symptoms. Pain relief was administered in the described case. A multidisciplinary intervention with palliative and oncological input is necessary. Current therapy options are not curative and the patient should only participate in a clinical trial if it does not affect their quality of life ${ }^{[4]}$.

\section{REFERENCES}

1. Turgut B. Ocular masquerade syndrome. Adv Ophthalmol Vis Syst 2018;8:148-149.

2. Pham CM, Kalyam K, Smith BT, Floyd J, Holds JB. Acute angle closure precipitated by hemorrhage and necrosis of a large uveal melanoma in the setting of systemic immunomodulatory therapy. Ocul Oncol Pathol 2017;3(4):254-258.

3. Oellers P, Wolkow N, Jakobiec FA, Kim IK. Hemorrhagic choroidal melanoma. Am J Ophthalmol Case Rep 2018;10:105-107.

4. Krantz BA, Dave N, Komatsubara KM, Marr BP, Carvajal RD. Uveal melanoma: epidemiology, etiology, and treatment of primary disease. Clin Ophthalmol 2017;11:279-289.

5. Singh AD, Medina CA, Singh N, Aronow ME, Biscotti CV, Triozzi PL. Fine-needle aspiration biopsy of uveal melanoma: outcomes and complications. Br J Ophthalmol 2016;100(4):456-462. 